Canad. Math. Bull. Vol. 20 (2), 1977

\title{
ON INTEGRAL ABEL-TYPE AND LOGARITHMIC METHODS OF SUMMABILITY
}

\author{
BY \\ E. C. HEAGY AND B. L. R. SHAWYER*
}

1. Introduction. In this paper, we define an integral logarithmic method of summability, extending the integral Abel-type methods defined by Jakimovski [6]. We examine the behaviour of the product of this method with integral Hausdorff methods. A full scale of strict inclusions for integral Abel-type methods is obtained and the integral logarithmic method is placed in this scale. For the analogous theorems for sequence-to-sequence Abel-type and logarithmic methods, see Borwein [1], [2].

We use the notation $M_{1} \supseteq M_{2}$ to mean that any sequence or function summable by the method $M_{2}$ is also summable by the method $M_{1}$ to the same limit. If $M_{1} \supseteq M_{2}$ and $M_{2} \supseteq M_{1}$, then we write $M_{1} \simeq M_{2}$. The notation $M_{1}=M_{2}$ indicates that the methods are equal; that is, their transforms are the same.

Throughout this paper, we require that $f$ be a real function which is bounded and Borel-measurable on every finite interval $[0, X]$. We shall suppose that $\sigma$ is real, that $\alpha>0$, that $\gamma>0$, and that $\beta>-1$.

2. Integral Hausdorff methods of summability. Integral (or continuous) Hausdorff methods of summability have been defined by Rogosinski [8] (see also [6]).

Let $\chi$ be a function of bounded variation on $[0,1]$. We extend $\chi$ to the entire real line by defining $\chi(t)=\chi(0)$ for $t<0$, and $\chi(t)=\chi(1)$ for $t>1$. For $y>0$, let $\mathbf{H}_{x}(y)=\mathbf{H}_{f ; x}(y)=\int_{0}^{1} f(y t) d \chi(t)$, where the integral is the Lebesgue-Stieltjes integral over $[0,1]$. Under our assumptions, $\mathbf{H}_{x}(y)$ exists for all $y>0$. If $\mathbf{H}_{\boldsymbol{x}}(\boldsymbol{y}) \rightarrow \sigma$ as $y \rightarrow \infty$, then we say that $f$ is $\mathbf{H}_{\boldsymbol{x}}$-summable to $\sigma$, and write $f(x) \rightarrow \sigma\left(\mathbf{H}_{x}\right)$.

We may assume that for $0<t<1, \chi(t)=\frac{1}{2}\{\chi(t-)+\chi(t+)\}$, and that $\chi(0)=0$. Hence each integral Hausdorff method corresponds to a unique $\chi$. Such a $\chi$ also generates a sequence-to-sequence Hausdorff method which we denote by $H_{x}$ (see, for example, [5, Ch. XI]). The conditions for the regularity of the integral and of the sequence-to-sequence Hausdorff methods are identical ([7], [8]; see also [5, Ch. XI]): $\mathbf{H}_{\chi}\left(\right.$ or $\left.H_{\chi}\right)$ is regular if and only if $\chi(0+)=\chi(0)$ and $\chi(1)-\chi(0)=1$.

Received by the editors February 5, 1976.

* Supported in part by the National Research Council of Canada. 
Certain other properties of the familiar sequence-to-sequence Hausdorff methods are also found in their functional analogues. For example, we have ([8]; cf. [7] or [5, Thm. 197]) $\mathbf{H}_{x} \mathbf{H}_{\psi}=\mathbf{H}_{\psi} \mathbf{H}_{x}$.

For $n=1,2, \ldots$, the moments of order $n$ of $\mathbf{H}_{\chi}$ (or of $H_{\chi}$ ) are given by $\mu_{n}=\int_{0}^{1} t^{n} d \chi(t)$. Rogosinski [8] has proved the following result.

THEOREM A. If $H_{x} \supseteq H_{\psi}$ and at most finitely many of the moments of $H_{\psi}$ vanish, then $\mathbf{H}_{\chi} \supseteq \mathbf{H}_{\psi}$.

3. Integral Cesàro-type methods. A specific class of integral Hausdorff methods is the integral Cesàro-type methods (see, for example, [2]). For $y>0$, let

$$
C_{\alpha, \beta}(y)=C_{f ; \alpha, \beta}(y)=\frac{\Gamma(\alpha+\beta+1)}{\Gamma(\alpha) \Gamma(\beta+1)} \int_{0}^{1}(1-t)^{\alpha-1} t^{\beta} f(y t) d t .
$$

If $C_{\alpha, \beta}(y) \rightarrow \sigma$ as $y \rightarrow \infty$, then we write $f(x) \rightarrow \sigma(C, \alpha, \beta)$. This is a regular summability method. The integral Cesàro method $(C, \alpha)$ is defined to be $(C, \alpha, 0)$ (see, for example, [5, p. 110] or [8]). The sequence-to-sequence analogues are well known [4], [5, Ch. V]. It is easy to show (using results in [4] and Theorem A) that $(C, \alpha, \beta) \simeq(C, \alpha)$, that $(C, \alpha, \gamma)(C, \gamma)=(C, \alpha+\gamma)$, and that $(C, \alpha+\gamma) \supseteq(C, \gamma)$, all analogues of results in [4].

4. Integral Abel-type summability. Jakimovski [6] has defined an integral Abel-type method of summability: for $\lambda>-1$ and $y>0$, let

$$
A_{\lambda}(y)=A_{f ; \lambda}(y)=\frac{1}{\Gamma(\lambda+1)} \int_{0}^{\infty} e^{-x} x^{\lambda} f(x y) d x .
$$

If $A_{\lambda}(y)$ exists as a Cauchy-Lebesgue integral for all $y>0$ and if $A_{\lambda}(y) \rightarrow \sigma$ as $y \rightarrow \infty$, then we say that $f$ is $A_{\lambda}$-summable to $\sigma$ and we write $f(x) \rightarrow \sigma\left(A_{\lambda}\right)$.

We note that the case $\lambda=0$ gives the Laplace transform. The method $A_{\lambda}$ is regular. It is the integral analogue of the sequence-to-sequence Abel-type method defined by Borwein [2], which generalizes the well-known Abel method.

The following three results are all due to Jakimovski [6].

THEOREM B. Let $\mathbf{H}_{x}$ be a regular integral Hausdorf method and let $\lambda>-1$. Then $A_{\lambda} \mathbf{H}_{x} \supseteq A_{\lambda}$.

Theorem C. Let $\lambda>\mu>-1$ and $y>0$. If $A_{f ; \lambda}(y)$ exists, then $A_{f ; \mu}(y)=$ $C_{\lambda-\mu, \mu} A_{f ; \lambda}(y)$.

Theorem D. For $\lambda>\mu>-1, A_{\mu} \supseteq A_{\lambda}$.

We now show that the inclusion in Theorem $\mathrm{D}$ is strict.

TheOREM 1. (cf. [2]) Let $\lambda>-1$. Then there exists a function which is 
$A_{\mu}$-summable to 0 for all $\mu$ satisfying $\lambda>\mu>-1$, but which is not $A_{\lambda}$ summable.

Proof. For all real $x$, we define $f(x)=\sum_{n=0}^{\infty} a_{n} x^{2 n}$, where $a_{n}=$ $(-1)^{n} \Gamma(\lambda+1) /(2 n+1) ! \Gamma(\lambda+2 n+2)$. It is easy to show that $A_{f ; \lambda}(y)=\sin y$. Thus $f$ is not $A_{\lambda}$-summable.

Suppose now that $\lambda>\mu>-1$. Using theorem $C$ and the Riemann-Lebesgue theorem, it now follows that

$$
\begin{aligned}
& A_{f ; \mu}(y)=C_{\lambda-\mu, \mu} A_{f ; \lambda}(y)=\frac{\Gamma(\lambda+1)}{\Gamma(\lambda-\mu) \Gamma(\mu+1)} \int_{0}^{1}(1-t)^{\lambda-\mu-1} t^{\mu} \sin (y t) d t \\
& \rightarrow 0 \quad \text { as } \quad y \rightarrow \infty ;
\end{aligned}
$$

that is, $f(x) \rightarrow 0\left(A_{\mu}\right)$.

5. The method $A_{-1}$. We now define an integral logarithmic method of summability which may be regarded as an extension of the integral Abel-type method $A_{\lambda}$ to the case $\lambda=-1$. Accordingly, we use the notation $A_{-1}$ to denote this method. For $y>0$, let

$$
A_{-1}(y)=A_{f ;-1}(y)=\frac{1}{\log (1+y)} \int_{0}^{\infty} \frac{e^{-(x+1) / y}}{x+1} f(x) d x .
$$

If $A_{-1}(y)$ exists as a Lebesgue integral for all $y>0$ and if $A_{-1}(y) \rightarrow \sigma$ as $y \rightarrow \infty$ then we write $f(x) \rightarrow \sigma\left(A_{-1}\right)$. This is a regular integral method, analogous to a sequence-to-function method given by Hardy [5, p. 81] (see also [1]).

We remark that changing the function $f$ on any finite interval does not affect its $A_{-1}$-summability.

Lemma. (cf. [1]) Let $\delta$ be real and let $g(x)=f(x) /(x+\delta)$ for $x \geq|\delta|+1$ and zero otherwise. If $f(x) \rightarrow \sigma\left(A_{-1}\right)$, then $g(x) \rightarrow 0\left(A_{-1}\right)$.

Proof. Let $M>|\delta|+1$ be constant. Let $\phi(t)=\int_{M}^{\infty} f(x) e^{-t(x+\delta)} /(x+1) d x$. Then we have that $\{\log (1+1 / t)\}^{-1} \phi(t) \rightarrow \sigma$ as $t \rightarrow 0+$. Hence there exists a constant $K$ such that for all $t$ in $(0,1),|\phi(t)| \leq K|\log (1+1 / t)|$. We also obtain that, as $t \rightarrow 0+$,

$$
\begin{aligned}
A_{g ;-1}(1 / t) & \sim \frac{e^{-t(1-\delta)}}{\log \left(1+\frac{1}{t}\right)} \int_{M}^{\infty} \frac{e^{-t(x+\delta)}}{(x+\delta)(x+1)} f(x) d x \\
& =\frac{e^{-t(1-\delta)}}{\log \left(1+\frac{1}{t}\right)} \int_{t}^{\infty} d z \int_{M}^{\infty} \frac{e^{-z(x+\delta)}}{(x+1)} f(x) d x \sim \frac{1}{\log \left(1+\frac{1}{t}\right)} \int_{t}^{\infty} \phi(z) d z .
\end{aligned}
$$


Let $\varepsilon \in(0,1)$ be given. Then for $0<t<\varepsilon$ we have that

$$
\frac{1}{\log \left(1+\frac{1}{t}\right)} \int_{\varepsilon}^{\infty} \phi(z) d z \rightarrow 0 \quad \text { as } \quad t \rightarrow 0+
$$

and

$$
\left|\frac{1}{\log \left(1+\frac{1}{t}\right)} \int_{t}^{\varepsilon} \phi(z) d z\right| \leq \frac{K}{\log \left(1+\frac{1}{t}\right)} \int_{t}^{\varepsilon} \log \left(1+\frac{1}{t}\right) d z \leq K \varepsilon
$$

Thus, for any $\varepsilon>0$, we have that $\lim \sup _{t \rightarrow 0+}\left|A_{g ;-1}(1 / t)\right| \leq K \varepsilon$, where $K$ is independent of $\varepsilon$. Thus it follows that $g(x) \rightarrow 0\left(A_{-1}\right)$.

Lemma. (cf. [1]) Let $\delta$ be real. Then $f(x) \rightarrow \sigma\left(A_{-1}\right)$ if and only if $f(x+\delta) \rightarrow$ $\sigma\left(A_{-1}\right)$.

Proof. Let $g(x)=f(x+\delta)$. Suppose that $f(x) \rightarrow \sigma\left(A_{-1}\right)$. From the previous lemma, it follows that

$$
A_{g ;-1}(y) \sim \frac{e^{\delta / y}}{\log (1+y)} \int_{|\delta|+1}^{\infty} \frac{e^{-(x+1) / y}}{(x+1)}\left(1+\frac{\delta}{x+1-\delta}\right) f(x) d x \rightarrow \sigma \quad \text { as } \quad y \rightarrow \infty ;
$$

that is, $f(x+\delta) \rightarrow \sigma\left(A_{-1}\right)$. Since $\delta$ is arbitrary, the result follows.

To investigate the product of integral Hausdorff methods and the method $A_{-1}$, we use a result due to Borwein [1].

TheOREM E. Let $\mathbf{H}_{x}$ be a regular integral Hausdorff method. For $x \geq 0$, let $g(x)$ be a continuous function. If $g(x) \rightarrow \sigma$ as $x \rightarrow \infty$, then

$$
\{\log (1+y)\}^{-1} \int_{0}^{1} \log (1+y t) g(y t) d \chi(t) \rightarrow \sigma \quad \text { as } \quad y \rightarrow \infty .
$$

TheOREM 2. (cf. [1]) Let $\mathbf{H}_{x}$ be a regular integral Hausdorff method. Then $A_{-1} \mathbf{H}_{\chi} \supseteq A_{-1}$.

Proof. Suppose that $f(x) \rightarrow \sigma\left(A_{-1}\right)$. We may further suppose that $f(x)=0$ for $x \leq 1$. Hence we have that

$$
\begin{aligned}
A_{-1} \mathbf{H}_{\chi}(y) & \sim\{\log (1+y)\}^{-1} \int_{1}^{\infty} \frac{e^{-u / y}}{u} d u \int_{0}^{1} f(u t) d \chi(t) \\
& =\{\log (1+y)\}^{-1} \int_{0}^{1} \log (1+y t) g(y t) d \chi(t),
\end{aligned}
$$

where

$$
g(x)=\{\log (1+x)\}^{-1} \int_{1}^{\infty} \frac{e^{-u / x}}{u} f(u) d u
$$


Using an argument as in [10, p. 181], we can show that $g$ is continuous. The desired result now follows from Theorem $\mathrm{E}$.

6. A scale of inclusions for integral Abel-type methods. We shall now place the $A_{-1}$ method in our scale of inclusions for integral Abel-type methods. From here on, we assume that $A_{\lambda}(y)$ exists as a Lebesgue integral.

Theorem 3. (cf. [1]) For $\lambda>-1, A_{-1} \supseteq A_{\lambda}$, and the inclusion is strict.

Before proving this theorem, we must develop some machinery.

THEOREM F. ([7], [3]) Let $c_{0}$ be a real constant. If $F(s)$ is an analytic function of $s=\rho+i r$ in the region $\rho>c_{0}$, and if there is a constant $K$ such that $\int_{-\infty}^{\infty}|F(c+i t)|^{2} d t<K$ for all $c>c_{0}$, then $F(s)=\int_{0}^{1} t^{s} \phi(t) d t$ for $\rho>c_{0}$, where $t^{c} \phi(t)$ is Lebesgue integrable on $[0,1]$ for all $c>c_{0}$.

Lemma. (cf. [3]) For $\lambda \geq 1,(x /(x+1))^{\lambda}-1=\int_{0}^{1} t^{x} \phi(t) d t$, where $t^{c} \phi(t)$ is Lebesgue integrable on $[0,1]$ for all $c>0$.

Proof. Setting $F(s)=(s /(s+1))^{\lambda}-1$, it is easy to show that, for $c>0$, $|F(c+i t)| \leq \lambda /\left\{(c+1)^{2}+t^{2}\right\}^{1 / 2}$, and further that $\int_{-\infty}^{\infty}|F(c+i t)|^{2} d t \leq \lambda^{2} \int_{-\infty}^{\infty} d t / 1+t^{2}$. The result now follows from Theorem $F$.

By adapting a method defined by Watson [9, p. 41], we define a method $J_{\lambda}$ as follows. For $y>0$ and $\lambda>-1$, let

$$
J_{\lambda}(y)=J_{f ; \lambda}(y)=\frac{1}{y^{\lambda} \log (1+y)} \int_{0}^{y}(y-x)^{\lambda} e^{-1 / x} x^{-1} f(x) d x .
$$

If $J_{\lambda}(y)$ exists for all $y>0$ and if $J_{\lambda}(y) \rightarrow \sigma$ as $y \rightarrow \infty$, then we say that $f$ is $J_{\lambda}$-summable to $\sigma$, and write $f(x) \rightarrow \sigma\left(J_{\lambda}\right)$.

Lemma. (cf. $[9, \S 4.6])$ Suppose that $\lambda>-1$. For $x>0$, let $g(x)=$ $(x /(x+1))^{\lambda} f(x)$. If $A_{\lambda}$ can be applied to $f$, then $J_{\lambda} A_{f ; \lambda}=A_{g ;-1}$.

Proof. For $y>0$, we have that

$$
\begin{aligned}
J_{\lambda} A_{f ; \lambda}(y) & =\frac{1}{y^{\lambda} \log (1+y)} \int_{0}^{y} \frac{(y-x)^{\lambda} e^{-1 / x}}{\Gamma(\lambda+1) x^{\lambda+2}} d x \int_{0}^{\infty} e^{-u / x} u^{\lambda} f(u) d u \\
& =\frac{1}{\log (1+y)} \int_{0}^{\infty} \frac{e^{-(u+1) / y}}{u+1}\left(\frac{u}{u+1}\right)^{\lambda} f(u) d u=A_{g ;-1}(y) .
\end{aligned}
$$

By letting $f(x)=1$ in the above, we see that $J_{\lambda}$ is regular, and further that the following result is true.

LEMMA. Let $\lambda>-1$. If $f(x) \rightarrow \sigma\left(A_{\lambda}\right)$, then $(x /(x+1))^{\lambda} f(x) \rightarrow \sigma\left(A_{-1}\right)$.

The next two lemmas extend this result.

Lemma. (cf. [3]) Let $\lambda \geq 1$. If $f(x) \rightarrow \sigma\left(A_{-1}\right)$, then $(x /(x+1))^{\lambda} f(x) \rightarrow \sigma\left(A_{-1}\right)$. 
Proof. Write $g(x)=(x /(x+1))^{\lambda} f(x)$. Without loss of generality, assume that $f(x)=0$ for $x<1$ and that $\sigma=0$. By an earlier lemma, we have that $(x /(x+1))^{\lambda}-$ $1=\int_{0}^{1} t^{x} \phi(t) d t$, where $t^{c} \phi(t)$ is Lebesgue integrable on $[0,1]$ for all $c>0$. Thus $(z /(x+1))^{\lambda}-1=\int_{0}^{\infty} e^{-x / z} \psi(z) d z$, where $\psi(z)=z^{-2} \phi\left(e^{-1 / z}\right)$. We note that $\psi(z)$ is Lebesgue integrable on $[\varepsilon, \infty)$ for any $\varepsilon>0$. In particular we have that $\int_{1}^{\infty}|\psi(z)| d z<\infty$. It now follows that

$$
\begin{aligned}
A_{g ;-1}(y) \sim & \frac{1}{\log (1+y)} \int_{1}^{\infty} u^{-1} e^{-u / y}\left(\frac{u}{u+1}\right)^{\lambda} f(u) d u \\
= & \frac{1}{\log (1+y)} \int_{1}^{\infty} u^{-1} e^{-u / y} f(u) d u \int_{0}^{\infty} e^{-u / z} \psi(z) d z \\
& +\frac{1}{\log (1+y)} \int_{1}^{\infty} u^{-1} e^{-u / y} f(u) d u .
\end{aligned}
$$

The second integral is $A_{f ;-1}(y)$, which, by assumption, tends to zero as $y \rightarrow \infty$.

Let $Y$ be a constant greater than one, and which will be specified later. Then for $y>Y$, we have that

$$
\begin{aligned}
A_{g ;-1}(y) & \sim \frac{1}{\log (1+y)}\left(\int_{0}^{Y}+\int_{Y}^{\infty}\right) \psi(z) d z \int_{1}^{\infty} u^{-1} e^{-u\left(y^{-1}+z^{-1}\right)} f(u) d u \\
& =I_{1}+I_{2}, \text { say. }
\end{aligned}
$$

It is easy to show that $I_{1} \rightarrow 0$ as $y \rightarrow \infty$. Further, since $y>y z /(y+z) \geq Y / 2$, we obtain

$$
\begin{aligned}
\left|I_{2}\right| & \leq \int_{Y}^{\infty} \frac{|\psi(z)|}{\log (1+y z /(y+z))} d z\left|\int_{1}^{\infty} u^{-1} e^{-u\left(y^{-1}+z^{-1}\right)} f(u) d u\right| \\
& \leq \sup _{x \geq Y / 2}\left|\frac{1}{\log (1+x)} \int_{1}^{\infty} u^{-1} e^{-u / x} f(u) d u\right| \cdot \int_{Y}^{\infty}|\psi(z)| d z \\
& \leq \sup _{x \geq Y / 2}\left|A_{f ;-1}(x)\right| \cdot \int_{1}^{\infty}|\psi(z)| d z .
\end{aligned}
$$

Since $Y$ can be chosen sufficiently large, the result now follows.

Lemma. Suppose that $\lambda>-1$. Then $f(x) \rightarrow \sigma\left(A_{-1}\right)$ if and only if $(x /(x+1))^{\lambda} f(x) \rightarrow \sigma\left(A_{-1}\right)$.

Proof.

(i) Necessity. Suppose that $f(x) \rightarrow \sigma\left(A_{-1}\right)$. For $\lambda \geq 1$, the preceding lemma gives the result. For $0 \leq \lambda<1$, we observe that

$$
\begin{aligned}
\left(\frac{x}{x+1}\right)^{\lambda} f(x)=\left(\frac{x+1}{x}\right)\left(\frac{x}{x+1}\right)^{\lambda+1} f(x) & \\
= & \left(\frac{x}{x+1}\right)^{\lambda+1} f(x)+\left(\frac{x}{x+1}\right)^{\lambda+1} \frac{f(x)}{x} \rightarrow \sigma\left(A_{-1}\right),
\end{aligned}
$$

since $\lambda+1 \geq 1$. The result for $-1<\lambda<0$ follows in a similar manner. 
(ii) Sufficiency. Suppose that $(x /(x+1))^{\lambda} f(x) \rightarrow \sigma\left(A_{-1}\right)$. For $-1<\lambda<1$, we note that $f(x)=(x /(x+1))^{-\lambda} \cdot(x /(x+1))^{\lambda} f(x) \rightarrow \sigma\left(A_{-1}\right)$, by the necessity part of this lemma, since $-\lambda>-1$. Suppose now that $\lambda \geq 1$. Then we have that $\lambda=\lambda_{0}+\lambda_{1}$, where $\lambda_{0}$ is a positive integer and $-1<\lambda_{1} \leq 0$. It now follows that

$$
\begin{aligned}
\left(\frac{x}{x+1}\right)^{\lambda_{1}} f(x) & =\left(\frac{x+1}{x}\right)^{\lambda_{0}}\left(\frac{x}{x+1}\right)^{\lambda} f(x) \\
& =\left(1+\frac{\lambda_{0}}{x}+\cdots+\frac{1}{x^{\lambda_{0}}}\right) \cdot\left(\frac{x}{x+1}\right)^{\lambda} f(x) \rightarrow \sigma\left(A_{-1}\right) .
\end{aligned}
$$

By the first part of the sufficiency, we obtain that $f(x) \rightarrow \sigma\left(A_{-1}\right)$, since $-1<\lambda_{1} \leq 0$.

By combining the results of this section with Theorem D and Theorem 1, we can now obtain a full scale of strict inclusions for integral Abel-type methods of summability.

\section{REFERENCES}

1. D. Borwein, A logarithmic method of summability, J. London Math. Soc., 33 (1958), 212-220.

2. D. Borwein, On a scale of Abel-type summability methods, Proc. Cambridge Philos. Soc., 53 (1957), 318-322.

3. D. Borwein, On methods of summability based on integral functions, II, Proc. Cambridge Philos. Soc., 56 (1960), 125-131.

4. D. Borwein, Theorems on some methods of summability, Quart. J. Math. (Oxford Ser.), 9 (1958), 310-316.

5. G. H. Hardy, Divergent Series, Oxford, Oxford, 1949.

6. A. Jakimovski (A. Amir), Some remarks on Tauberian theorems, Quart. J. Math. (Oxford Ser.), 9 (1958), 114-131.

7. W. W. Rogosinski, On Hausdorff's methods of summability, Proc. Cambridge Philos. Soc., 38 (1942), 166-192.

8. W. W. Rogosinski, On Hausdorf's methods of summability, II, Proc. Cambridge Philos. Soc., 38 (1942), 344-363.

9. B. B. Watson, Tauberian theorems on a scale of Abel-type summability methods, Ph.D. Thesis, University of Western Ontario, 1974.

10. D. V. Widder, The Laplace Transform, Princeton University Press, Princeton, 1946.

Scarborough College,

UNIVERSITY OF TORONTO,

1265 Military Trail,

West Hill, Ontario, Canada,

M1C $1 \mathrm{~A} 4$.

Department of MATHEMATics,

UNIVERSITY OF WESTERN ONTARIO,

London, Ontario, Canada,

N6A $5 B 9$. 